\title{
Oil palm defensin: a thermal stable peptide that restricts the mycelial growth of Ganoderma boninense
}

\begin{abstract}
Plant defensins are plant defence peptides that have many different biological activities, including antifungal, antimicrobial, and insecticidal activities. A cDNA (EgDFS) encoding defensin was isolated from Elaeis guineensis. The open reading frame of EgDFS contained 231 nucleotides encoding a 71-amino acid protein with a predicted molecular weight at 8.69 $\mathrm{kDa}$, and a potential signal peptide. The eight highly conserved cysteine sites in plant defensins were also conserved in EgDFS. The EgDFS sequence lacking 30 amino acid residues at its N-terminus (EgDFSm) was cloned into Escherichia coli BL21 (DE3) pLysS and successfully expressed as a soluble recombinant protein. The recombinant EgDFSm was found to be a thermal stable peptide which demonstrated inhibitory activity against the growth of G. boninense possibly by inhibiting starch assimilation. The role of EgDFSm in oil palm defence system against the infection of pathogen G. boninense was discussed.
\end{abstract}

Keyword: Defensin; Oil palm; Elaeis guineensis; Ganoderma; Pathogen; $\alpha$-amylase 